\title{
Reduced low frequency noise in electron beam evaporated MgO magnetic tunnel junctions
}

\author{
Z. Diao, J. F. Feng, ${ }^{\text {a) }}$ H. Kurt, G. Feng, and J. M. D. Coey \\ CRANN and School of Physics, Trinity College, Dublin 2, Ireland
}

(Received 7 April 2010; accepted 27 April 2010; published online 18 May 2010)

\begin{abstract}
We compare low frequency noise in magnetic tunnel junctions with $\mathrm{MgO}$ barriers prepared by electron-beam evaporation with those prepared by radiofrequency sputtering, both showing a high tunneling magnetoresistance. The normalized noise parameter in the parallel state of junctions with evaporated barriers is at least one order of magnitude lower than that in junctions with sputtered barriers, and exhibits a weaker bias dependence. The lowest normalized noise is in the $10^{-11} \mu \mathrm{m}^{2}$ range. A lower density of oxygen vacancies acting as charge trap states in the evaporated $\mathrm{MgO}$ is responsible for the lower noise. (C) 2010 American Institute of Physics. [doi:10.1063/1.3431620]
\end{abstract}

Magnetic tunnel junctions (MTJs) have attracted a great deal of attention since the demonstration of the tunneling magnetoresistance (TMR) effect at room temperature. ${ }^{1,2}$ Following theoretical predictions, ${ }^{3,4}$ a large TMR ratio of up to $200 \%$ at room temperature in MTJs with CoFeB electrodes and $\mathrm{MgO}$ tunnel barriers was achieved. ${ }^{5,6} \mathrm{~A}$ record room temperature TMR of $604 \%$ has since been reported in a pseudospin valve stack, ${ }^{7}$ which is close to the theoretical maximum. ${ }^{3,4}$ Major advances in MTJs have led to important applications in hard-disk read heads, ${ }^{8}$ sensors, ${ }^{9}$ and magnetic random access memory. ${ }^{10}$ For magnetic field sensing applications, both signal and noise in the MTJ are of equal significance. It has been found previously that $1 / f$ noise usually dominates other kinds of noise, e.g., thermal noise and shot noise, at frequencies up to a few kilohertz in an MTJ device, and it limits the low-frequency sensitivity. ${ }^{11-16}$ The $1 / f$ noise in these devices is normally quantified by the Hooge parameter $\alpha$, defined as $A f S_{V} / V^{2}$, in which $A$ is the junction area, $f$ the frequency, $S_{V}$ the noise power spectrum density, and $V$ the voltage applied to the MTJ. ${ }^{12,17}$ In conventional radio frequency (rf) sputtered MTJs with CoFeB electrodes and $\mathrm{MgO}$ tunnel barriers, $\alpha$ was reported to decrease as a function of annealing time and reach a minimum value of $2 \times 10^{-10} \mu \mathrm{m}^{2} .{ }^{18}$ The lowest $\alpha$ value ever observed in MTJs, between $(1-5) \times 10^{-11} \mu \mathrm{m}^{2}$, was achieved in fully epitaxial $\mathrm{Fe}(100) / \mathrm{MgO}(100) / \mathrm{Fe}(100)$ junctions grown by molecular beam epitaxy (MBE). ${ }^{19}$

Recently, some of us suggested electron-beam evaporation as an alternative to MBE or rf-sputtering for growing high quality $\mathrm{MgO}$ tunnel barriers. The junctions fabricated in this way have been shown to possess high TMR ratios of $\sim 240 \%$ at room temperature and a reduced density of oxygen vacancies, compared to counterparts grown entirely by rf-sputtering. ${ }^{20}$ In this work, we report on low frequency noise in the MTJs with electron-beam evaporated $\mathrm{MgO}$ barriers of different thicknesses (EB-MTJs), comparing it to that measured in our MTJs with rf-sputtered $\mathrm{MgO}$ tunnel barriers (rf-MTJs). We show that the Hooge parameter $\alpha$ in the EB-MTJs in their parallel state is at least one order of magnitude lower than that in the rf-MTJs, across a large range of resistance-area (RA) product $5 \times 10^{2}<\mathrm{RA}<1$

${ }^{a)}$ Electronic mail: jfeng@tcd.ie. $\times 10^{6} \Omega \mu \mathrm{m}^{2}$, when all the other layers in the stacks are grown in the same way.

The MgO-based MTJ stack with a layer sequence Ta $5 / \mathrm{Ru} 30 / \mathrm{Ta} 5 / \mathrm{Ni}_{81} \mathrm{Fe}_{19} 5 / \mathrm{Ir}_{22} \mathrm{Mn}_{78} 10 / \mathrm{Co}_{90} \mathrm{Fe}_{10} 2.5 /$

$\mathrm{Ru} 0.9 / \mathrm{Co}_{40} \mathrm{Fe}_{40} \mathrm{~B}_{20}(\mathrm{CoFeB}) 3 / \mathrm{MgO} t / \mathrm{CoFeB} 3 / \mathrm{Ta} 5 / \mathrm{Ru} 10$ (thicknesses in nanometer) was grown on thermally oxidized Si wafers (Fig. 1 inset). All metallic layers were deposited by dc-magnetron sputtering in a Shamrock sputtering tool. For the EB-MTJs, the MgO layers with $t=1.5,2.0$, and $2.5 \mathrm{~nm}$ were grown by electron-beam evaporation in a separate ultrahigh vacuum chamber of the Shamrock system having a base pressure of $2.1 \times 10^{-10}$ Torr, after deposition of the bottom electrodes in the $\mathrm{HV}$ chamber (base pressure $1 \times 10^{-7}$ Torr). The wafer was then transferred back to the $\mathrm{HV}$ chamber to complete the stack. For rf-MTJs, $\mathrm{MgO}$ with $t=1.2-3.5 \mathrm{~nm}$ was grown by $\mathrm{rf}$ sputtering from two $\mathrm{MgO}$ targets in a target-facing-target gun, in another chamber of the Shamrock system having a base pressure of about $1 \times 10^{-8}$ Torr. All MTJ layers were deposited without breaking the vacuum during the growth process. The stack was patterned into microscale MTJs with an area ranging from 3 to $1150 \mu \mathrm{m}^{2}$, using either electron-beam or UV lithography. High vacuum postannealing of the devices was performed in the temperature range of $325-425^{\circ} \mathrm{C}$ in an applied mag-

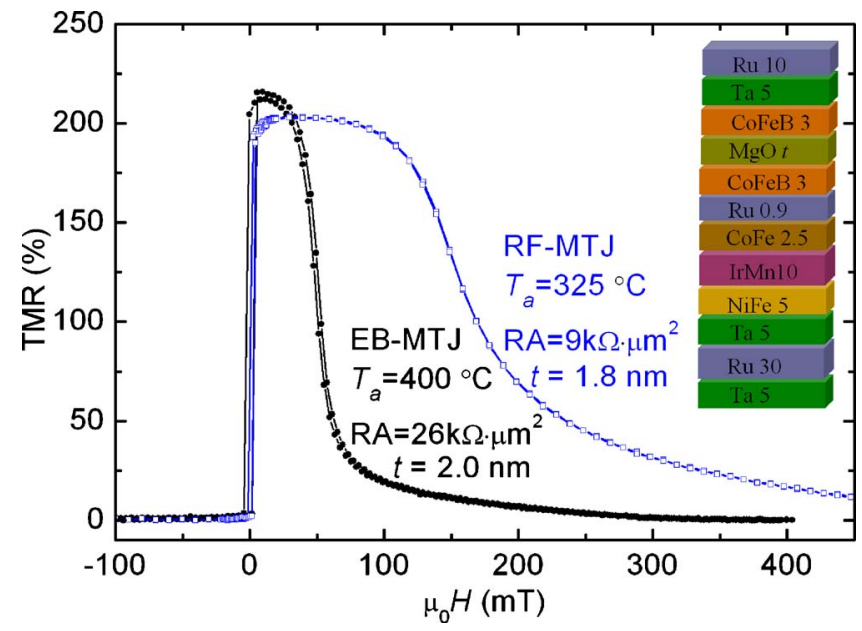

FIG. 1. (Color online) MR curves for an EB-MTJ and a rf-MTJ. Inset is a sketch of the multilayer stack investigated in this work, where thicknesses are in nanometers; $1.2<t<3.5$. 


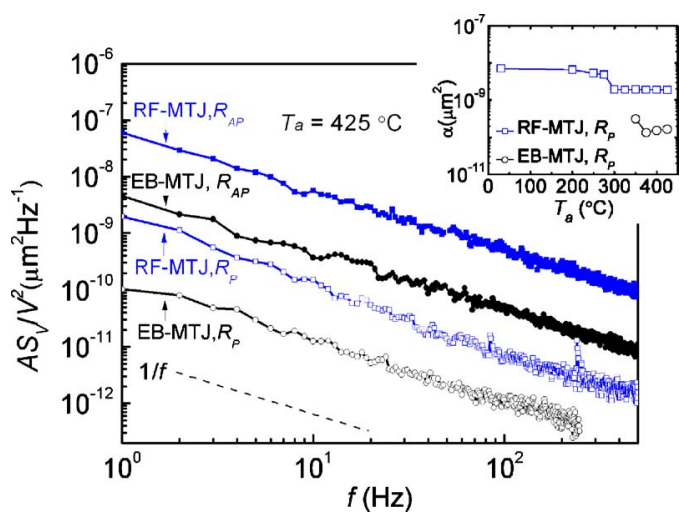

FIG. 2. (Color online) The normalized power spectral density of the noise as a function of frequency for the EB- and rf-MTJ for the parallel and antiparallel states. The thermal noise and amplifier noise have been subtracted. Inset shows the evolution of the Hooge parameter of the rf-MTJs and EBMTJs in their parallel state as a function of annealing temperature.

netic field of $800 \mathrm{mT}$ for $1 \mathrm{~h}$. All magnetotransport and noise measurements were performed by a four-probe method at room temperature. Positive bias is defined as the direction of electrons tunneling from the free to the pinned $\mathrm{CoFeB}$ layer. Further details on the sample growth process and measurement can be found in Refs. 20-22.

Figure 1 shows MR curves for an EB-MTJ and a rfMTJ. The TMR ratios are $212 \%$ and $203 \%$, and their RA values are $9 \mathrm{k} \Omega \mu \mathrm{m}^{2}$ and $26 \mathrm{k} \Omega \mu \mathrm{m}^{2}$, respectively. We note here that our EB-MTJs show a similar TMR ratio to the rf-MTJs but the RA is greater for the EB junctions with a given barrier thickness. The high magnetoresistance reflects the high quality of our $\mathrm{MgO}$ barriers of both types. Figure 2 plots the normalized noise power spectral density $\left(A S_{V} / V^{2}\right)$ as a function of frequency for the EB- and rf-MTJs. The amplifier noise and thermal noise have been subtracted from the measured $S_{V}$. We only show the noise power spectral density under low current bias, to avoid the complication of bias dependence of $\alpha$. A $1 / f$ low frequency noise is observed for either the parallel or antiparallel state for both types of MTJs but the noise in the antiparallel state is always higher.

It is evident in Fig. 2 that our EB-MTJ possesses a much lower normalized noise power spectrum in the parallel state compared to the rf-MTJ, despite the fact that all the other layers in the stack are grown in exactly the same way. Low frequency noise in MTJs in the parallel state is thought to be dominated by barrier noise. ${ }^{18}$ Our results, therefore, indicate that the electron-beam evaporated $\mathrm{MgO}$ barrier is quieter than the rf sputtered one.

We note that the normalized noise in the parallel state of an MTJ decreases with thermal annealing, and reaches its minimum after annealing at $300{ }^{\circ} \mathrm{C}$ for an hour. Further increase in the annealing temperature does not cause much decrease in the MTJs' normalized noise (Fig. 2 inset), although the magnetoresistance continues to increase after. This observation supports a recent report of Stearret et al., ${ }^{18}$ who found a similar behavior of low frequency noise in MTJs with sputtered $\mathrm{MgO}$ tunnel barriers as a function of annealing time. All the annealing temperatures we used in this study are above $300{ }^{\circ} \mathrm{C}$.

We summarize the Hooge parameter for the parallel state as a function of RA in the EB- and rf-MTJs in Fig. 3(a). It is found that $\alpha$ of the rf-MTJs first falls with decreasing RA, then reaches a roughly constant value $\left(\sim 10^{-9} \mu \mathrm{m}^{2}\right)$ in de-

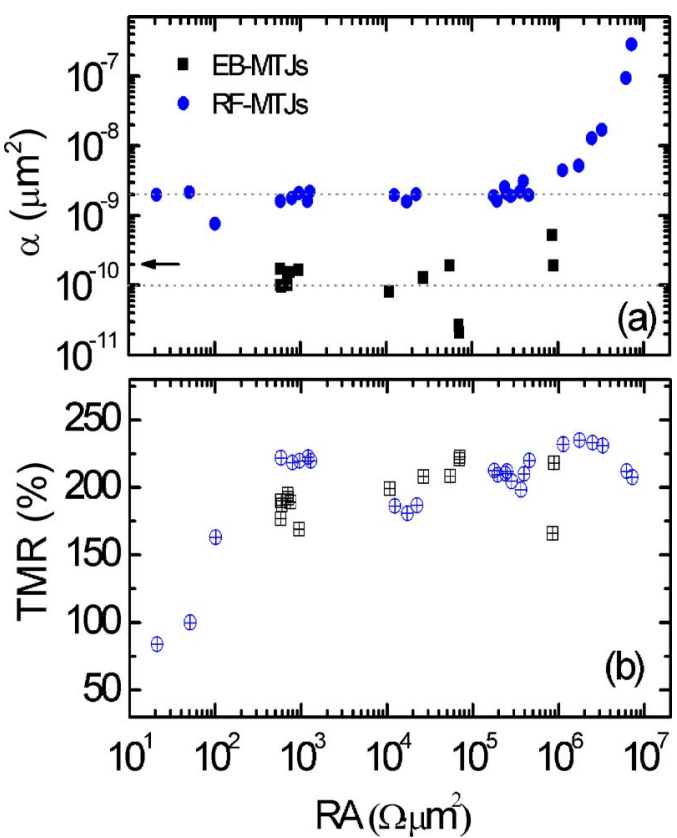

FIG. 3. (Color online) The RA dependence of the Hooge paremeter $(\alpha)$ (a) and TMR (b) for two types of MTJs. The arrow on the left indicates the minimum noise level reported in Ref. 18. Lines are guides to the eye.

vices with RA below $5 \times 10^{5} \Omega \mu \mathrm{m}^{2}$. This is similar to the behavior summarized by Gokce et al. ${ }^{23}$ for low frequency noise in MTJs fabricated in different laboratories. This can possibly be related to Hooge's original idea that $1 / f$ noise scales inversely as the number of mobile charge carriers in the active area of the device. ${ }^{24}$ The $\alpha$ values in EB-MTJs are of order $10^{-10} \mu \mathrm{m}^{2}$ when RA is lower than $10^{6} \Omega \mu \mathrm{m}^{2}$, which is at least one order of magnitude lower than those in rf-MTJs. The lowest $\alpha$ measured is $2.1 \times 10^{-11} \mu \mathrm{m}^{2}$ for a MTJ with the RA of $7 \times 10^{4} \Omega \mu \mathrm{m}^{2}$. This is comparable to that reported in fully epitaxial single crystal $\mathrm{Fe} / \mathrm{MgO} / \mathrm{Fe}$ MTJs. ${ }^{19}$ We also observe that $\alpha$ in EB-MTJs shows more scatter than that in rf-MTJs. This can probably be attributed to the fact that a lower $\alpha$ value is more sensitive to the local variation in the barrier quality. ${ }^{19}$

Figure 3(b) shows the corresponding TMR ratio for both types of MTJs. The TMR ratio varies very little when RA exceeds $10^{3} \Omega \mu \mathrm{m}^{2}$. Similar observations have been previously reported in Ref. 25 . The highest TMR values are $240 \%$ and $235 \%$ for the EB- and rf-MTJs, respectively. We see no correlation between TMR and $\alpha$. The large difference of $\alpha$ for the parallel state of the two types of MTJs, shown in Fig. 3(a), cannot be explained by a difference in their TMR ratio.

Figure 4 shows the bias dependence of $\alpha$ for the EB- and rf-MTJs for the parallel and antiparallel states. The Hooge parameter $\alpha$ for the antiparallel state is higher than for the parallel state due to magnetic fluctuations which contribute largely to the low frequency noise. In either state, the $\alpha$ value remains roughly constant when the bias voltage is below 100 $\mathrm{mV}$ for both types of MTJs. However, $\alpha$ for the antiparallel state shows a strong bias dependence at greater voltages for both types of MTJs. This can be linked to the nonlinear dependence of junction resistance on bias voltage. ${ }^{18}$ In the parallel state, $\alpha$ in the rf-MTJ shows a slight decrease when a bias voltage larger than $200 \mathrm{mV}$ is applied, as reported in Refs. 17 and 23, while $\alpha$ in the EB-MTJ is nearly bias independent. The increase in $\alpha$ at high positive bias for the rf- 


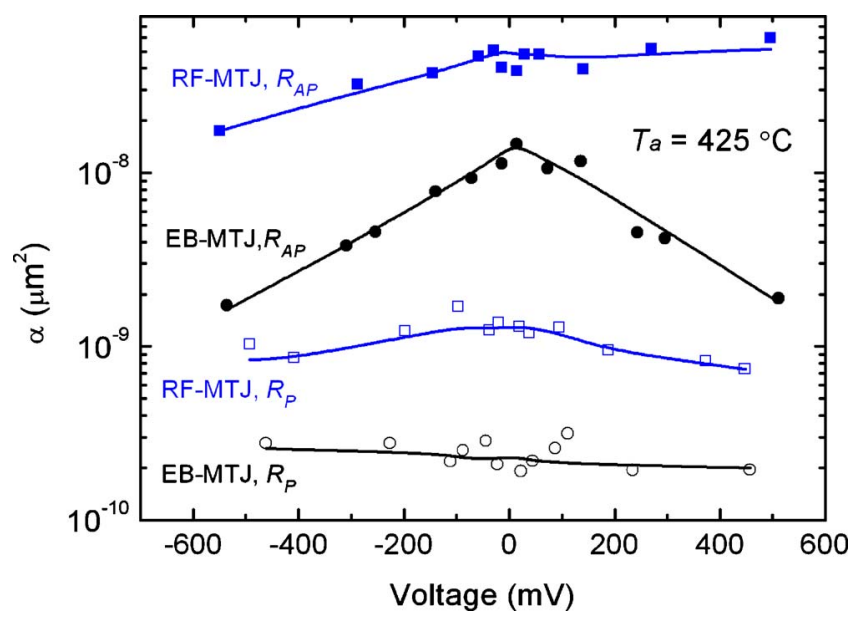

FIG. 4. (Color online) The bias dependence of the Hooge parameter $\alpha$ for two types of MTJs at parallel and antiparallel states. Lines are guides to the eye.

MTJ in its antiparallel state is related to the appearance of random telegraph noise (not shown).

It is found that the tunnel barrier and the barrier/ electrode interfaces can be improved during the annealing process, which leads to a high TMR due to crystallization of $\mathrm{CoFeB}$ electrodes and improved quality of the $\mathrm{MgO}$ barrier. In our case, the CoFeB layers in both types of MTJs are expected to be quite similar, as they were both grown by sputtering and underwent exactly the same annealing treatment. The difference of $\alpha$ must largely originate from the $\mathrm{MgO}$ barrier due to the different oxide growth methods. It has been shown that upon annealing, the rf- $\mathrm{MgO}$ retains a larger $\mathrm{d}$-spacing, while the evaporated $\mathrm{MgO}$ has the same d-spacing as bulk $\mathrm{MgO} .{ }^{20}$ A higher density of oxygen vacancies forms in the $\mathrm{MgO}$ layer near the two interfaces for the rf-MTJs, while the density of oxygen vacancies in the $\mathrm{MgO}$ barrier is much lower for EB-MTJs, due to the fact that deposition of $\mathrm{MgO}$ by electron-beam evaporation leads to almost layer-by-layer growth. ${ }^{20}$ Although the quality of the $\mathrm{MgO}$ tunnel barrier can be greatly improved during the annealing process, the oxygen vacancies cannot be completely removed. They act as charge traps which create low frequency noise. This explains why the $\alpha$ value in our EB-MTJs is much lower, and supports the conclusion of other authors ${ }^{18}$ that sputtered MTJs have an apparent noise floor due to a particular defect type which has to be eliminated by an improved fabrication process.

In conclusion, a lower $1 / f$ noise, characterized by the Hooge parameter $\alpha$, obtained in EB-MTJs compared to that found in rf-MTJs is attributed to the absence of charge trap states in the $\mathrm{MgO}$ tunnel barrier associated with oxygen vacancies. Electron-beam evaporation is an alternative way to produce high quality $\mathrm{MgO}$ tunnel barriers with noise performance comparable to that found for fully epitaxial single crystalline junctions grown by MBE. ${ }^{19}$ It is faster and more cost effective, and it could be easily integrated in industrial production. TMR-based magnetic field sensors with electronbeam evaporated $\mathrm{MgO}$ barriers offer about an order of magnitude improvement in their signal-to-noise ratio compared to those with conventional sputtered $\mathrm{MgO}$ tunnel barriers.

This work was supported by Science Foundation Ireland as part of the MANSE Project No. 05/IN/1850. Some support was also provided by Enterprise Ireland, as part of the "Spincurrents" FoNE network. The authors would like to thank Professor E. R. Nowak for help with the noise measurement setup.

${ }^{1}$ T. Miyazaki and N. Tezuka, J. Magn. Magn. Mater. 139, L231 (1995).

${ }^{2}$ J. S. Moodera, L. R. Kinder, T. M. Wong, and R. Meservey, Phys. Rev. Lett. 74, 3273 (1995)

${ }^{3}$ W. H. Butler, X.-G. Zhang, T. C. Schulthess, and J. M. MacLaren, Phys. Rev. B 63, 054416 (2001).

${ }^{4}$ J. Mathon and A. Umersky, Phys. Rev. B 63, 220403 (2001)

${ }^{5}$ S. S. P. Parkin, C. Kaiser, A. Panchula, P. M. Rice, B. Hughes, M. Samant, and S.-H. Yang, Nature Mater. 3, 862 (2004).

${ }^{6}$ S. Yuasa, T. Nagahama, A. Fukushima, Y. Suzuki, and K. Ando, Nature Mater. 3, 868 (2004).

${ }^{7}$ S. Ikeda, J. Hayakawa, Y. Ashizawa, Y. M. Lee, K. Miura, H. Hasegawa, M. Tsunoda, F. Matsukura, and H. Ohno, Appl. Phys. Lett. 93, 082508 (2008).

${ }^{8}$ S. Yuasa and D. D. Djayaprawira, J. Phys. D 40, R337 (2007).

${ }^{9}$ W. Shen, B. D. Schrag, M. J. Carter, and G. Xiao, Appl. Phys. Lett. 93, 033903 (2008).

${ }^{10}$ W. J. Gallagher and S. S. P. Parkin, IBM J. Res. Dev. 50, 5 (2006).

${ }^{11}$ J. Scola, H. Polovy, C. Fermon, M. Pannetier-Lecoeur, G. Feng, K. Fahy, and J. M. D. Coey, Appl. Phys. Lett. 90, 252501 (2007).

${ }^{12}$ E. R. Nowak, M. B. Weissman, and S. S. P. Parkin, Appl. Phys. Lett. 74, 600 (1999).

${ }^{13}$ L. Jiang, E. R. Nowak, P. E. Scott, J. Johnson, J. M. Slaugher, J. J. Sun, and R. W. Dave, Phys. Rev. B 69, 054407 (2004).

${ }^{14}$ C. Ren, X. Y. Liu, B. D. Schrag, and G. Xiao, Phys. Rev. B 69, 104405 (2004).

${ }^{15}$ D. Mazumdar, X. Y. Liu, B. D. Schrag, M. Carter, W. F. Shen, and G. Xiao, Appl. Phys. Lett. 91, 033507 (2007).

${ }^{16}$ F. Guo, G. McKusky, and E. Dan Dahlberg, Appl. Phys. Lett. 95, 062512 (2009).

${ }^{17}$ J. M. Almeida, P. Wisniowski, and P. P. Freitas, IEEE Trans. Magn. 44, 2569 (2008).

${ }^{18}$ R. Stearrett, W. G. Wang, L. R. Shah, A. Gokce, J. Q. Xiao, and E. R. Nowak, J. Appl. Phys. 107, 064502 (2010).

${ }^{19}$ F. G. Aliev, R. Guerrero, D. Herranz, R. Villar, F. Greullet, C. Tiusan, and M. Hehn, Appl. Phys. Lett. 91, 232504 (2007).

${ }^{20}$ H. Kurt, K. Oguz, T. Niizeki, and J. M. D. Coey, J. Appl. Phys. 107, 083920 (2010).

${ }^{21}$ J. F. Feng, G. Feng, J. M. D. Coey, X. F. Han, and W. S. Zhan, Appl. Phys. Lett. 91, 102505 (2007).

${ }^{22}$ J. F. Feng, Z. Diao, G. Feng, E. R. Nowak, and J. M. D. Coey, Appl. Phys. Lett. 96, 052504 (2010).

${ }^{23}$ A. Gokce, E. R. Nowak, S. H. Yang, and S. S. P. Parkin, J. Appl. Phys. 99, 08A906 (2006).

${ }^{24}$ F. N. Hooge, Phys. Lett. A 29, 139 (1969).

${ }^{25}$ J. Hayakawa, S. Ikeda, F. Mastsukura, H. Takahashi, and H. Ohno, Jpn. J. Appl. Phys., Part 2 44, L587 (2005). 